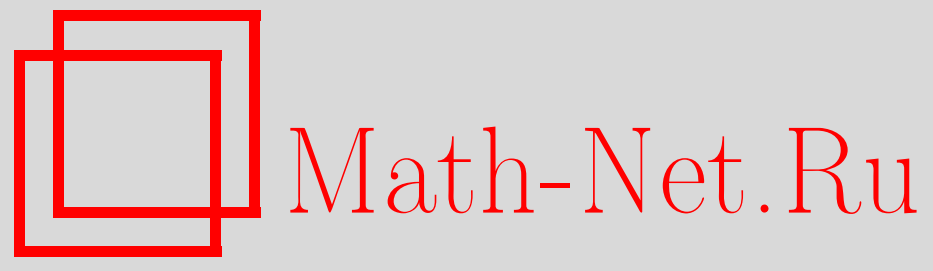

Г. Б. Сизых, Расщепление уравнений НавьеСтокса для одного класса осесимметричных течений, Вестн. Сам. гос. техн. ун-та. Сер. Физ.мат. науки, 2020, номер 1, 163-173

DOI: https://doi.org/10.14498/vsgtu1740

Использование Общероссийского математического портала MathNet.Ru подразумевает, что вы прочитали и согласны с пользовательским соглашением

http: //www . mathnet.ru/rus/agreement

Параметры загрузки:

IP : 3.81 .55 .215

26 апреля 2023 г., 14:11:16

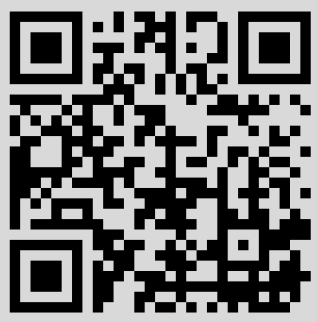


Вестн. Сам. гос. техн. ун-та. Сер. Физ.-мат. науки. 2020. Т. 24, № 1. С. $163-173$ ISSN: 2310-7081 (online), 1991-8615 (print) dol https://doi.org/10.14498/vsgtu1740

УДК 532.5.032

\title{
Расщепление уравнений Навье-Стокса для одного класса осесимметричных течений
}

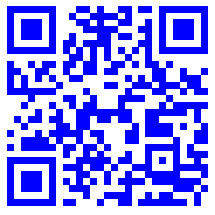

\section{Г. Б. Сизых}

Московский авиационный институт (национальный исследовательский университет), Россия, 125993, Москва, Волоколамское шоссе, 4.

\begin{abstract}
Аннотация
В рамках уравнений Навье-Стокса рассмотрены нестационарные осесимметричные течения однородной вязкой несжимаемой жидкости, в которых осевая и окружная скорости зависят только от радиуса и от времени, а радиальная скорость равна нулю. Показано, что скорость таких течений представляет собой сумму скоростей двух течений вязкой несжимаемой жидкости: осевого течения (радиальная и окружная скорости равны нулю) и окружного течения (радиальная и осевая скорости равны нулю). Осевое и окружное движения происходят независимо, не оказывая никакого взаимного влияния. Это позволяет расщеплять краевые задачи для рассматриваемого типа течений, содержащие три неизвестные функции (давление, окружная и осевая скорости), на две задачи, каждая из которых содержит две неизвестные функции (давление и одна из компонент скорости). При этом сумма давлений осевого и окружного течений будет давлением исходного течения. Обнаруженная возможность расщепления позволяет с использованием известных решений пополнить «запасы» осевых и окружных точных решений. Эти решения, в свою очередь, можно суммировать в различных комбинациях и в результате получать скорости и давления новых точных решений уравнений Навье-Стокса.
\end{abstract}

Ключевые слова: вязкая несжимаемая жидкость, расщепление уравнений Навье-Стокса, точные решения.

Получение: 22 августа 2019 г. / Исправление: 15 октября 2019 г. / Принятие: 11 ноября 2019 г. / Публикация онлайн: 2 апреля 2020 г.

\footnotetext{
Научная статья

(2) (1) Контент публикуется на условиях лицензии Creative Commons Attribution 4.0 International (https://creativecommons.org/licenses/by/4.0/deed.ru)

Образец для цитирования

С изы х Г. Б. Расщепление уравнений Навье-Стокса для одного класса осесимметричных течений // Вестн. Сам. гос. техн. ун-та. Сер. Физ.-мат. науки, 2020. Т. 24, № 1. С. 163173. doi: $10.14498 /$ vsgtu1740.

\section{Сведения об авторе}

Григорий Борисович Сизых (1) https://orcid.org/0000-0001-5821-8596 кандидат физико-математических наук, доцент; доцент; каф. прикладной математики; e-mail: 010203@yandex.ru
} 
Введение. В конце двадцатого века в результате развития вычислительной техники и вычислительных методов доминирующими способами исследования течений жидкости стали численные расчеты. При этом, как свидетельствуют обзоры $[1,2]$, «запас» точных решений уравнений Навье-Стокса продолжал пополняться еще активнее, чем в первой половине двадцатого века $[3,4]$. Это связано в первую очередь с тем, что сходимость большинства численных методов доказана только для линеаризованных уравнений (что оставляет сомнение в правильности расчетов задач с нелинейными уравнениями). Поэтому численные алгоритмы верифицируют, используя точные решения (см., например, [5]). Заметим, что единственная известная в настоящее время альтернатива верификации находится на начальной стадии развития. Речь идет о методах обнаружения ошибки в расчетах, для которых неизвестны точные решения. В таких методах [6-8] проверяются некоторые следствия уравнений Навье-Стокса, и решение признается ошибочным, если в нем нарушены эти следствия. Однако не исключено, что эти следствия окажутся выполненными на ошибочном решении, и ошибка останется незамеченной. Поэтому верификация, основанная на сравнении с точным решением, до сих пор остается актуальной. Кроме того, интерес к точным решениям связан с тем, что с их помощью можно оценивать погрешность асимптотических теорий (см. например, [9]). Точные решения также используются в теоретических исследованиях в качестве примеров течений с теми или иными свойствами. Например, в [10] приведен пример задачи, имеющей неединственное решение, а в $[11,12]$ - примеры течений с определенными свойствами завихренности.

Таким образом, точные решения уравнений Навье-Стокса востребованы как в доминирующей в настоящее время вычислительной гидродинамике, так и в теоретической гидродинамике. Это объясняет появление новых решений $[10,11,13-17]$ и после обзоров [1,2]. В 1919 году Тркал показал, как из винтового (завихренность параллельна скорости) решения уравнения Эйлера получить нестационарное решение уравнений Навье-Стокса (способ Тркала описан, например, в [16]). Поэтому к числу новых решений уравнений НавьеСтокса, полученных в последние годы, можно отнести и новые винтовые решения уравнения Эйлера $[18,19]$. Данная статья также посвящена точным решениям уравнений Навье-Стокса. В ней предлагается способ получения новых решений с использованием известных решений.

В данной статье рассматривается частный случай осесимметричных течений вязкой несжимаемой жидкости - течения, в которых радиальная компонента скорости равна нулю, а остальные компоненты скорости зависят только от расстояния до оси симметрии и от времени. При этом давление может зависеть еще и от осевой координаты. Линии тока в этих течениях лежат на цилиндрах, осью которых является ось симметрии течения. Практическая значимость этих течений состоит в том, что они могут описывать движение жидкости в длинных цилиндрических трубах круглого сечения. Поэтому для краткости будем называть такие течения $r t \nu$-течениями в цилиндре. Исполь-

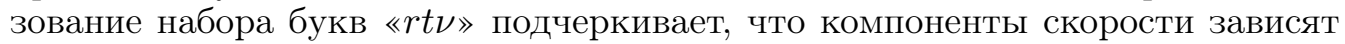
только от переменных $r$ и $t$ и что речь идет именно о вязких течениях (греческой буквой « $\nu »$ обычно обозначают вязкость). Самыми известными $r t \nu$ течениями в цилиндре являются (осевое) течение Пуазейля [20] и два осесимметричных окружных течения: течение между двумя вращающимися цилиндрами с непроницаемыми стенками и течение Озеена (процесс диффузии вихря) [21]. Известны и другие точные решения для $r t \nu$-течений в цилиндре. 
Так, например, в монографии [22] упоминаются точные решения для двух

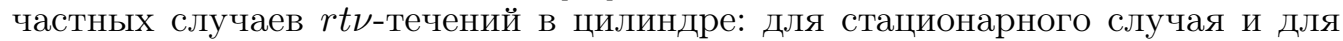
случая нулевой осевой скорости (вращение жидкости вокруг оси). В обзоpe [1] можно найти точные решения для трех частных случаев $r t \nu$-течений в цилиндре: для случая нулевой осевой скорости, для стационарного случая при нулевой окружной скорости (течение пуазейлевского типа) и отдельно для нестационарного случая при нулевой окружной скорости (результат статьи [23]). Некоторые точные решения для $r t \nu$-течений в цилиндре можно найти в обзоре [2], в частности, окружное нестационарное течение Тейлоpa. В статье [11] представлено несколько $r t \nu$-течений в цилиндре в качестве примеров течений с определенными свойствами завихренности. Наиболее широкий класс точных решений для $r t \nu$-течений в цилиндре представлен в статье [3], в которой, по утверждению автора обзора [4], «дана сводка известных точных решений» по состоянию на 1936 год. В статье [3] сначала показано, что точное решение можно получить, если окружную и радиальную скорости вычислить по некоторым (полученным в [3]) формулам через производные по переменным $r$ и $t$ от любой функции $\psi(r, t)$, удовлетворяющей уравнению $\nu \Delta \Delta \psi=\partial \Delta \psi / \partial t$, где $\Delta$ - оператор Лапласа. Затем получены некоторые решения этого уравнения. В результате получился достаточно широкий класс точных решений уравнений Навье-Стокса. Однако даже если найти все решения уравнения $\nu \Delta \Delta \psi=\partial \Delta \psi / \partial t$, то и тогда предложенный в [3] подход не позволит получить все возможные точные решения для $r t \nu$-течений в цилиндре. Причина этого заключается в том, что исследование $r t \nu$-течений в цилиндре в статье [3] проведено без учета их специфических особенностей - на основе формул, верных для любых осесимметричных течений. Эти формулы представляют не все решения уравнений, полученных после исключения давления из уравнений Навье-Стокса (после ротации векторного уравнения Навье-Стокса), а только некоторые из этих решений.

Исключение давления приводит к уравнениям с производными компонент скорости белее высокого порядка, чем порядок этих производных в уравнениях Навье-Стокса. И «платой» за уменьшение количества неизвестных функций (исключение давления) является повышение порядка производных. В данной статье для $r t \nu$-течений в цилиндре предлагается другой способ упрощения системы уравнений Навье-Стокса, без исключения давления, который основан на одном специфическом свойстве $r t \nu$-течений в цилиндре. Речь идет о том, что, как будет показано ниже, скорость $r t \nu$-течений в цилиндре представляет собой сумму скоростей двух течений вязкой несжимаемой жидкости, каждое из которых подчиняется уравнениям Навье-Стокса: осевого течения (радиальная и окружная скорости равны нулю) и окружного течения (радиальная и осевая скорости равны нулю). Это позволяет расщеплять краевые задачи для рассматриваемого типа течений, содержащие три неизвестные функции (давление, окружная и осевая скорости), на две задачи, каждая из которых содержит две неизвестные функции (давление и одна из компонент скорости). При этом сумма давлений осевого и окружного течений будет давлением исходного течения. Кроме того, ниже будет показано, что любые два $r t \nu$-течения в цилиндре допускают сложение скоростей, и в итоге получается скорость некоторого другого $r t \nu$-течения в цилиндре. В общем случае давление точного решения, полученного в результате такого сложения скоростей, не будет суммой исходных давлений, однако легко может быть рассчитано по полю окружной скорости путем взятия неопределенного интеграла. В итоге возможность суммирования превращает известный в на- 
стоящее время запас точных решений (в том числе решений, содержащихся в перечисленной выше литературе) в такой класс точных решений для $r t \nu$ течений в цилиндре, который содержит все известные решения и значительно превышает их совокупность по разнообразию.

1. Основные обозначения и уравнения движения. Течение однородной ньютоновской вязкой несжимаемой жидкости описывается уравнениями Навье-Стокса, в которых потенциал объемных сил и давление, отнесенное к плотности, входят в виде их суммы. Это обстоятельство позволяет легко пересчитывать точные решения для течений, происходящих в отсутствие объемных сил, в точные решения для течений в потенциальных полях. Поэтому ограничимся рассмотрением течений, в которых отсутствуют объемные силы.

Пусть осесимметричное течение однородной ньютоновской вязкой несжимаемой жидкости происходит в отсутствие объемных сил. Введем следующие безразмерные переменные: $\boldsymbol{V}-$ скорость; $\boldsymbol{\Omega}=\operatorname{rot} \boldsymbol{V}$ - завихренность; $p$ - давление, отнесенное к плотности, которое ниже для краткости будем называть давлением; Re - число Рейнольдса. Движение жидкости описывается уравнениями Навье-Стокса, которые можно представить в форме

$$
\begin{gathered}
\frac{\partial \boldsymbol{V}}{\partial t}+\boldsymbol{\Omega} \times \boldsymbol{V}=-\frac{1}{\operatorname{Re}} \operatorname{rot} \boldsymbol{\Omega}-\nabla\left(p+\frac{\boldsymbol{V}^{2}}{2}\right), \\
\operatorname{div} \boldsymbol{V}=0 .
\end{gathered}
$$

Пусть далее $\operatorname{Or} \varphi z$ - цилиндрическая система координат с началом в точке $O$ (ось $O z$ совпадает с осью симметрии течения). Обозначим через $\boldsymbol{e}_{r}, \boldsymbol{e}_{\varphi}$, $\boldsymbol{e}_{z}$ - правую тройку единичных векторов в радиальном, окружном и осевом направлениях соответственно. Скорость $\boldsymbol{V}$ может быть представлена в виде $\boldsymbol{V}=V_{r} \boldsymbol{e}_{r}+V_{\varphi} \boldsymbol{e}_{\varphi}+V_{z} \boldsymbol{e}_{z}$

Рассмотрим $r t \nu$-течения в цилиндре, то есть течения, в которых $V_{r} \equiv 0$, а компоненты скорости $V_{\varphi}$ и $V_{z}$ зависят только от радиуса $r$ и от времени $t$ и не зависят от окружной и от осевой координат $\varphi$ и $z$. При этом давление $p$ может зависеть не только от $r$ и $t$, но еще и от осевой координаты $z$. Скорость $\boldsymbol{V}$ и давление $p$ таких течений представляется в виде

$$
\boldsymbol{V}=V_{\varphi}(r, t) \boldsymbol{e}_{\varphi}+V_{z}(r, t) \boldsymbol{e}_{z}, \quad p=p(r, z, t) .
$$

Наряду с этим будем рассматривать еще два особых типа $r t \nu$-течений в цилиндре: окружное и осевое. Окружное $r t \nu$-течение в цилиндре - это течение, в котором $\boldsymbol{V}=V_{\varphi}(r, t) \boldsymbol{e}_{\varphi}$. Осевое $r t \nu$-течение в цилиндре - это течение, в котором $\boldsymbol{V}=V_{z}(r, t) \boldsymbol{e}_{z}$ (течение пуазейлевского типа).

Уравнение неразрывности (2) в цилиндрической системе координат имеет вид

$$
\frac{1}{r} \frac{\partial}{\partial r}\left(r V_{r}\right)+\frac{1}{r} \frac{\partial}{\partial \varphi} V_{\varphi}+\frac{\partial}{\partial z} V_{z}=0
$$

Если учесть, что $V_{r} \equiv 0$, а $V_{\varphi}$ и $V_{z}$ зависят только от переменных $r$ и $t$, то получается, что как скорость $\boldsymbol{V}=V_{\varphi} \boldsymbol{e}_{\varphi}+V_{z} \boldsymbol{e}_{z}$, так и скорости $\boldsymbol{V}_{\varphi}=V_{\varphi} \boldsymbol{e}_{\varphi}$ и $\boldsymbol{V}_{z}=V_{z} \boldsymbol{e}_{z}$ по отдельности удовлетворяют уравнению неразрывности (2). Поэтому ниже будем проверять только выполнение уравнения (1). И не будем всякий раз напоминать, что для $r t \nu$-течений в цилиндре уравнение неразрывности выполняется «автоматически».

2. Расщепление уравнений Навье-Стокса. Пусть $\boldsymbol{V}=V_{\varphi} \boldsymbol{e}_{\varphi}+V_{z} \boldsymbol{e}_{z}-$ скорость, а $p$ - давление некоторого $r t \nu$-течения в цилиндре. Это значит, что 
скорость $\boldsymbol{V}$ и давление $p$ удовлетворяют уравнению (1) и представляются в виде (3). Поскольку $V_{\varphi}$ и $V_{z}$ зависят только от переменных $r$ и $t$, векторное уравнение (1), записанное в цилиндрической системе координат, равносильно системе из трех скалярных уравнений:

$$
\begin{gathered}
\frac{V_{\varphi}^{2}}{r}=\frac{\partial p}{\partial r} \\
\frac{\partial V_{\varphi}}{\partial t}-\frac{1}{\operatorname{Re}} \frac{\partial}{\partial r}\left(\frac{1}{r} \frac{\partial\left(r V_{\varphi}\right)}{\partial r}\right)=0 \\
\frac{\partial V_{z}}{\partial t}-\frac{1}{\operatorname{Re}} \frac{1}{r} \frac{\partial}{\partial r}\left(r \frac{\partial V_{z}}{\partial r}\right)=-\frac{\partial p}{\partial z}
\end{gathered}
$$

Для $r t \nu$-течений в цилиндре левые части уравнений (4) и (5) не зависят от $z$. Поэтому

$$
\frac{\partial^{2} p}{\partial z \partial r}=\frac{\partial^{2} p}{\partial z^{2}}=0 .
$$

Следовательно, в $r t \nu$-течении в цилиндре давление можно представить в виде

$$
p=p(r, z, t)=\hat{p}(r, t)+z A(t) .
$$

Возможность представить давление в виде (6) позволяет расщепить уравнение (1) на систему уравнений для окружной скорости

$$
\left\{\begin{array}{l}
\frac{V_{\varphi}^{2}}{r}=\frac{\partial \hat{p}}{\partial r} \\
\frac{\partial V_{\varphi}}{\partial t}-\frac{1}{\operatorname{Re}} \frac{\partial}{\partial r}\left(\frac{1}{r} \frac{\partial\left(r V_{\varphi}\right)}{\partial r}\right)=0
\end{array}\right.
$$

и на уравнение для осевой скорости

$$
\frac{\partial V_{z}}{\partial t}-\frac{1}{\operatorname{Re}} \frac{1}{r} \frac{\partial}{\partial r}\left(r \frac{\partial V_{z}}{\partial r}\right)=-A(t) .
$$

Уравнения (7) и (8) по отдельности описывают не какие-то особые случаи окружного и осевого движений. Система уравнений (7) описывает самый общий случай окружного движения (в классе $r t \nu$-течений в цилиндре), а уравнение (8) - самый общий случай осевого движения. В итоге приходим к двум следующим результатам.

1. РАСЩЕПЛЕНИЕ УРАВНЕНИЙ НАВьЕ-СТОкСА. Скорость $\boldsymbol{V}=V_{\varphi} \boldsymbol{e}_{\varphi}+V_{z} \boldsymbol{e}_{z}$ и давление $p=p(r, z, t)=\hat{p}(r, t)+z A(t)$ любого $r t \nu$-течения в иилиндре представляются соответственно в виде суммы скоростей и давлений

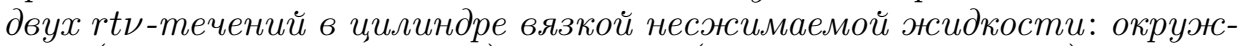
ного $\left(\boldsymbol{V}_{\varphi}=V_{\varphi} \boldsymbol{e}_{\varphi}, p=\hat{p}(r, t)\right)$ и осевого $\left(\boldsymbol{V}_{z}=V_{z} \boldsymbol{e}_{z}, p=z A(t)\right)$.

2. СУМмиРовАНИЕ ОКРУЖНЫХ и ОСЕВЫХ РЕШЕНИЙ. Сумма скоростей и давлений любого окружного и любого осевого rtv-течений в иилиндре вязкой несжимаемой жидкости является скоростъю и давлением неко-

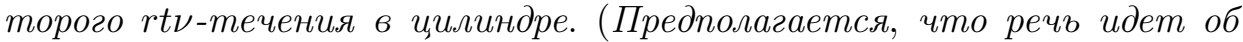
одной и той же жидкости, то есть, что в суммируемых течениях число Рейнольдса $\operatorname{Re}$ одинаково.) 
Эти утверждения справедливы как для стационарных, так и для нестационарных течений. Независимо от того, стационарной или нестационарной является окружная составляющая скорости течения, осевая составляющая также может быть как стационарной, так и нестационарной.

3. Обсуждение. Утверждение о возможности суммирования осевых и окружных решений представляется достаточно очевидным. Действительно, поскольку $\operatorname{rot} \boldsymbol{V}_{\varphi}$ имеет осевое направление, a $\operatorname{rot} \boldsymbol{V}_{z}-$ окружное направление, сумма двух уравнений Навье-Стокса

$$
\frac{\partial \boldsymbol{V}_{z}}{\partial t}+\operatorname{rot} \boldsymbol{V}_{z} \times \boldsymbol{V}_{z}=-\frac{1}{\operatorname{Re}} \operatorname{rot} \operatorname{rot} \boldsymbol{V}_{z}-\nabla\left(p_{z}+\frac{\boldsymbol{V}_{z}^{2}}{2}\right)
$$

и

$$
\frac{\partial \boldsymbol{V}_{\varphi}}{\partial t}+\operatorname{rot} \boldsymbol{V}_{\varphi} \times \boldsymbol{V}_{\varphi}=-\frac{1}{\operatorname{Re}} \operatorname{rot} \operatorname{rot} \boldsymbol{V}_{\varphi}-\nabla\left(p_{\varphi}+\frac{\boldsymbol{V}_{\varphi}^{2}}{2}\right)
$$

с учетом равенства нулю векторного произведения коллинеарных векторов дает уравнение Навье-Стокса для суммарного течения:

$$
\begin{aligned}
\frac{\partial\left(\boldsymbol{V}_{z}+\boldsymbol{V}_{\varphi}\right)}{\partial t}+\operatorname{rot}\left(\boldsymbol{V}_{z}+\boldsymbol{V}_{\varphi}\right) \times\left(\boldsymbol{V}_{z}+\boldsymbol{V}_{\varphi}\right)= & \\
& =-\frac{1}{\operatorname{Re}} \operatorname{rot} \operatorname{rot}\left(\boldsymbol{V}_{z}+\boldsymbol{V}_{\varphi}\right)-\nabla\left(p_{z}+p_{\varphi}+\frac{\boldsymbol{V}_{z}^{2}}{2}+\frac{\boldsymbol{V}_{\varphi}^{2}}{2}\right) .
\end{aligned}
$$

Доказанное в предыдущем разделе обратное утверждение (о расщеплении) не столь очевидно, поскольку из (1) неочевидно, например, что выражение

$$
\frac{\partial \boldsymbol{V}_{z}}{\partial t}+\operatorname{rot} \boldsymbol{V}_{z} \times \boldsymbol{V}_{z}+\frac{1}{\operatorname{Re}} \operatorname{rot} \operatorname{rot} \boldsymbol{V}_{z}
$$

является градиентом какой-либо функции. Поэтому для доказательства утверждения о расщеплении пришлось воспользоваться не векторной, а координатной записью уравнений Навье-Стокса.

Решения уравнений Навье-Стокса для $r t \nu$-течений в цилиндре будем называть $r t \nu$-решениями в цилиндре. Из формул (7) и (8) следует, что допускается сложение $r t \nu$-решений в цилиндре, в каждом из которых обе скорости $V_{\varphi}$ и $V_{z}$ могут быть не равны тождественно нулю. При этом «осевая составляющая» давления в новом решении $z A(t)$ будет суммой «осевых составляющих» давления исходных решений. Однако «окружная составляющая» давления в новом решении $\hat{p}(r, t)$ в общем случае не будет суммой «окружных составляющих» давления исходных решений. Величина $\hat{p}(r, t)$ для нового решения вычисляется через суммарную окружную скорость $V_{\varphi}=V_{\varphi}(r, t)$ по формуле

$$
\hat{p}(r, t)=\hat{p}_{0}+\int_{r_{0}}^{r} V_{\varphi}^{2}(\vartheta, t) \cdot \vartheta^{-1} d \vartheta
$$

где $\hat{p}_{0}>0$ и $r_{0} \geqslant 0$ - константы. 
Поскольку при суммировании точных $r t \nu$-решений в цилиндре получается (точное) выражение для суммарной окружной скорости $V_{\varphi}=V_{\varphi}(r, t)$, приведенный выше интеграл для любого значения $r$ может быть посчитан на компьютере с любой наперед заданной точностью (ровно так, как, например, это имеет место для синуса, косинуса, экспоненты, цилиндрических функций и т.п.). В этом смысле можно говорить о том, что при суммировании точных $r t \nu$-решений в цилиндре получается новое точное $r t \nu$-решение в цилиндре. В итоге обнаруженная выше возможность расщепления и суммирования превращает известный в настоящее время запас точных решений в источник получения новых решений. Всякое известное $r t \nu$-решение в цилиндре расщепляется, пополняя тем самым «запасы» осевых и окружных точных решений, суммирование которых в различных комбинациях позволяет получать новые точные решения.

Заключение. В рамках уравнений Навье-Стокса рассмотрены осесимметричные течения вязкой несжимаемой жидкости, в которых радиальная компонента скорости равна нулю, а окружная и осевая компоненты скорости не зависят от окружной и осевой координат (зависят только от расстояния до оси симметрии и от времени). При этом давление может зависеть еще и от осевой координаты. Такие течения предложено назвать $r t \nu$-течениями в цилиндре.

Показано, что скорость $r t \nu$-течения в цилиндре представляет собой сумму скоростей двух течений вязкой несжимаемой жидкости, каждое из которых подчиняется уравнениям Навье-Стокса: осевого течения (радиальная и окружная скорости равны нулю) и окружного течения (радиальная и осевая скорости равны нулю). Осевое и окружное движения происходят независимо друг от друга, не оказывая никакого взаимного влияния. Это позволяет расщеплять краевые задачи для рассматриваемого типа течений, содержащие три неизвестные функции (давление, окружная и осевая скорости), на две задачи, каждая из которых содержит две неизвестные функции (давление и одна из компонент скорости). При этом сумма давлений осевого и окружного течений будет давлением исходного течения.

Полученный результат также позволяет получать новые точные решения с использованием существующего «запаса» точных решений.

Конкурирующие интересы. Конкурирующих интересов не имею.

Авторский вклад и ответственность. Я несу полную ответственность за предоставление окончательной версии рукописи в печать. Окончательная версия рукописи мною одобрена.

Финансирование. Исследование выполнялось без финансирования.

Благодарность. Автор благодарен рецензенту за тщательное прочтение статьи и ценные предложения и комментарии.

\section{Библиографический список}

1. Drazin P. G., Riley N. The Navier-Stokes Equations: A Classification of Flows and Exact Solutions / London Mathematical Society Lecture Note Series. vol. 334. Cambridge: Cambridge Univ. Press, 2006. x+196 pp. doi: 10.1017/CB09780511526459.

2. Пухначев В. В. Симметрии в уравнениях Навье-Стокса // Успехи механики, 2006. № 6. C. 3-76.

3. Berker R. Sur quelques cas d'intégration des équations du movement d'un fluide visqueux incompressible. Paris-Lille: Imprimerie A. Taffin-Lefort, 1936. 
4. Neményi P. F. Recent developments in inverse and semi-inverse methods in the mechanics of continua// Adv. Appl. Mech., 1951. vol.2. pp. 123-151. doi: 10.1016/S0065-2156(08) 70300-4.

5. Власенко В. В., Волков А. В., Трошин А. И. Выбор метода аппроксимации вязких членов в методе Галеркина с разрывными базисными функциями // Ученые записки ЦАГИ, 2013. Т. 44, № 3. С. 18-38.

6. Голубкин В. Н., Марков В. В., Сизых Г. Б. Интегральный инвариант уравнений движения вязкого газа // ПMМ, 2015. Т. 79, №6. С. 808-816.

7. Vyshinsky V. V., Sizykh G. B. Verification of the calculation of stationary subsonic flows and presentation of results / Intern. Conf. on 50 years of the development of grid-characteristic method. GCM50 2018 / Smart Modeling for Engineering Systems, 133, 2019. pp. 228-235. doi : 10.1007/978-3-030-06228-6_19.

8. Vyshinsky V. V., Sizykh G. B. The verification of the calculation of stationary subsonic flows and the presentation of the results // Math. Models Comput. Simul., 2019. vol.11, no. 1. pp. 97-106. doi: 10.1134/S2070048219010162.

9. Петров А. Г. О точных и асимптотических решениях уравнений Навье-Стокса в слое жидкости между сближающимися и удаляющимися пластинами // Изв. РАН. МЖГ, 2014. № 2. С. 44-57.

10. Аристов С. Н., Князев Д. В. Трёхмерное струйное течение вязкой жидкости с плоскими свободными границами // Изв. РАН. МЖГ, 2017. № 2. С. 50-53. doi: 10.7868/ S0568528117020050.

11. Коцур О. С. О существовании локальных способов вычисления скорости переноса вихревых трубок с сохранением их интенсивности // Труды МФТИ, 2019. Т. 11, № 1. C. $76-85$.

12. Сизых Г. Б. Винтовые вихревые линии в осесимметричных течениях вязкой несжимаемой жидкости // ПMM, 2009. Т. 83, № 3. С. 370-376. doi : 10.1134/S0032823519030123.

13. Kumar M., Kumar R. On some new exact solutions of incompressible steady state Navier-Stokes equations // Meccanica, 2014. vol.49, no.2. pp. 335-345. doi:10.1007/ s11012-013-9798-4.

14. Артышев С. Г. Описание некоторых плоских вращающихся течений несжимаемой жидкости с помощью цилиндрических функций // ПММ, 2015. Т. 79, № 2. С. 236-241.

15. Аристов С. Н., Просвиряков Е. Ю. Нестационарные слоистые течения завихренной жидкости // Изв. РАН. МЖГ, 2016. № 2. С. 25-31.

16. Ковалев В. П., Просвиряков Е. Ю., Сизых Г. Б. Получение примеров точных решений уравнений Навье-Стокса для винтовых течений методом суммирования скоростей // Труды МФТИ, 2017. Т. 9, № 1. С. 71-88.

17. Weidman P. D., Mansur S., Ishak A. Biorthogonal stretching and shearing of an impermeable surface in a uniformly rotating fluid system // Meccanica, 2017. vol. 52, no. 7. pp. 1515-1525. doi: 10.1007/s11012-016-0507-y.

18. Верещагин В. П., Субботин Ю. Н., Черных Н. И. К механике винтовых потоков в идеальной несжимаемой невязкой сплошной среде / Тр. ИММ УрО РАН, Т. 18, 2012. C. $120-134$.

19. Верещагин В. П., Субботин Ю. Н., Черных Н. И. Некоторые решения уравнений движения для несжимаемой вязкой сплошной среды / Тр. ИММ УрО РАН, Т. 19, 2013. C. $48-63$.

20. Poiseuille J.-L.-M. Recherches expérimentales sur le mouvement des liquides dans les tubes de très-petits diamètres. Paris: Imprimerie Royale, 1844.

21. Batchelor G. K. An Introduction to Fluid Dynamics. Cambridge: Cambridge Univ. Press, 2000. xviii+615 pp. doi : 10.1017/СВ09780511800955.

22. Страхович К. И. Механика вязкой жидкости. І. Общая частъ. Л.: ЛГУ, 1940. 201 с.

23. Szymánski P. Quelques solutions exactes des équations d'hydrodynamique du fluide visqueux dans le cas d'un tube cylindrique // J. Math. Pures Appl., 1932. vol.11. pp. 67-108, http://eudml.org/doc/234063. 


\title{
MSC: 76D05, 76D99
}

\section{The splitting of Navier-Stokes equations for a class of axisymmetric flows}

\section{G. B. Sizykh}

Moscow Aviation Institute (National Research University),

4, Volokolamskoe shosse, Moscow, 125993, Russian Federation.

\begin{abstract}
In the framework of the Navier-Stokes equations, unsteady axisymmetric flows of a homogeneous viscous incompressible fluid, in which the axial and circumferential velocities depend only on radius and time are considered, and the radial velocity is zero. It is shown that the velocity of such flows is the sum of the velocities of two flows of a viscous incompressible fluid: axial flow (radial and circumferential velocities are zero) and circumferential flow (radial and axial velocities are zero). Axial and circumferential movements occur independently, without exerting any mutual influence. This allows us to split the boundary value problems for the type of flows under consideration containing three unknown functions (pressure, circumferential and axial velocities) into two problems, each of which contains two unknown functions (pressure and one of the velocity components). In this case, the sum of pressures of the axial and circumferential flow will be the pressure of the initial flow. The discovered possibility of splitting allows using known solutions to replenish the "reserves" of axial and circumferential exact solutions. These solutions, in its turn, can be summed in various combinations and, as a result, give the velocities and pressures of new exact solutions of the Navier-Stokes equations.
\end{abstract}

Keywords: viscous incompressible fluid, splitting of the Navier-Stokes equations, exact solutions.

Received: 22 ${ }^{\text {nd }}$ August, 2019 / Revised: $15^{\text {th }}$ October, $2019 /$

Accepted: $11^{\text {th }}$ November, $2019 /$ First online: $2^{\text {nd }}$ April, 2020

Competing interests. I have no competing interests.

Authors' contributions and responsibilities. I take full responsibility for submitting the final manuscript in print. I approved the final version of the manuscript.

Funding. The research has not had any funding.

\section{Research Article}

(ㅇ)(7) The content is published under the terms of the Creative Commons Attribution 4.0 International License (http://creativecommons.org/licenses/by/4.0/)

Please cite this article in press as:

S iz y kh G. B. The splitting of Navier-Stokes equations for a class of axisymmetric flows, Vestn. Samar. Gos. Tekhn. Univ., Ser. Fiz.-Mat. Nauki [J. Samara State Tech. Univ., Ser. Phys. Math. Sci.], 2020, vol. 24, no. 1, pp. 163-173. doi: 10.14498/vsgtu1740 (In Russian).

Author's Details:

Grigory B. Sizykh (10) https://orcid.org/0000-0001-5821-8596

Cand. Phys. \& Math. Sci; Associate Professor; Dept. of Applied Mathematics;

e-mail: o102o3@yandex.ru 
Acknowledgments. The author is grateful to the reviewer for careful reading of the paper and valuable suggestions and comments.

\section{References}

1. Drazin P. G., Riley N. The Navier-Stokes Equations: A Classification of Flows and Exact Solutions, London Mathematical Society Lecture Note Series, vol. 334. Cambridge, Cambridge Univ. Press, 2006, x+196 pp. doi : 10.1017/CB09780511526459.

2. Pukhnachev V V. Symmetries in Navier-Stokes equations, Uspehi Mehaniki, 2006, no. 6, pp. 3-76 (In Russian).

3. Berker R. Sur quelques cas d'intégration des équations du movement d'un fluide visqueux incompressible. Paris-Lille, Imprimerie A. Taffin-Lefort, 1936, http://eudml.org/doc/ 192863.

4. Neményi P. F. Recent developments in inverse and semi-inverse methods in the mechanics of continua, Adv. Appl. Mech., 1951, vol.2, pp. 123-151. doi:10.1016/S0065-2156(08) $70300-4$.

5. Troshin A.I., Vlasenko V. V, Wolkov A. V. Selection of viscous terms approximation in discontinuous galerkin method, TsAGI Science Journal, 2013, vol.44, no. 3, pp. 327-354. doi : 10.1615/TsAGISciJ . 2013009684.

6. Golubkin V. N., Markov V. V., Sizykh G. B. The integral invariant of the equations of motion of viscous gas, J. Appl. Math. Mech., 2015, vol.79, no.6, pp. 566-571. doi: 10 . 1016/j.jappmathmech.2016.04.002.

7. Vyshinsky V. V., Sizykh G. B. Verification of the calculation of stationary subsonic flows and presentation of results, In: Intern. Conf. on 50 years of the development of grid-characteristic method. GCM50 2018, Smart Modeling for Engineering Systems, 133, 2019, pp. 228-235. doi: 10.1007/978-3-030-06228-6_19.

8. Vyshinsky V. V., Sizykh G. B. The verification of the calculation of stationary subsonic flows and the presentation of the results, Math. Models Comput. Simul., 2019, vol.11, no. 1, pp. 97-106. doi: 10.1134/S2070048219010162.

9. Petrov A. G. Exact and asymptotic solutions of the Navier-Stokes equations in a fluid layer between plates approaching and moving apart from one another, Fluid Dyn., 2014, vol. 49, no. 2, pp. 174-187. doi : 10.1134/S0015462814020069.

10. Aristov S. N., Knyazev D. V. Three-dimensional viscous jet flow with plane free boundaries, Fluid Dyn., 2017, vol. 52, no. 2, pp. 215-218. doi: 10.1134/S0015462817020053.

11. Kotsur O. S. On the existence of local formulae of the transfer velocity of local tubes that conserve their strengths, Trudy MFTI, 2019, vol.11, no. 1, pp. 76-85 (In Russian).

12. Sizykh G. B. Helical vortex lines in axisymmetric viscous incompressible fluid flows, Fluid Dyn., 2019, vol.54, no. 3, pp. 1038-1042. doi : 10.1134/S0015462818060083.

13. Kumar M., Kumar R. On some new exact solutions of incompressible steady state Navier-Stokes equations, Meccanica, 2014, vol.49, no. 2, pp. 335-345. doi:10.1007/ s11012-013-9798-4.

14. Artyshev S. G. The description of several plane rotating flows of an incompressible fluid using cylindrical functions, J. Appl. Math. Mech., 2015, vol. 79, no. 2, pp. 159-163. doi: 10 . 1016/j.jappmathmech.2015.07.006.

15. Aristov S. N., Prosviryakov E. Yu. Unsteady layered vortical fluid flows, Fluid Dyn., 2016, vol. 51, no. 2, pp. 148-154. doi: 10.1134/S0015462816020034.

16. Kovalev V. P., Prosviryakov E. Yu., Sisykh G. B. Obtaining examples of exact solutions to Navier-Stokes equations for helical flows by method of summing velocities, Trudy MFTI, 2017, vol. 9, no. 1, pp. 71-88 (In Russian).

17. Weidman P. D., Mansur S., Ishak A. Biorthogonal stretching and shearing of an impermeable surface in a uniformly rotating fluid system, Meccanica, 2017, vol.52, no. 7, pp. 1515-1525. doi: 10.1007/s11012-016-0507-y. 
18. Vereshchagin V. P., Subbotin Yu. N., Chernykh N. I. On the mechanics of helical flows in an ideal incompressible nonviscous continuous medium, Proc. Steklov Inst. Math., 2014, vol. 284, pp. 159-174. doi: 10.1134/S008154381402014X.

19. Vereshchagin V. P., Subbotin Yu. N., Chernykh N. I. Some solutions of continuum equations for an incompressible viscous medium, Proc. Steklov Inst. Math., 2014, vol. 287, pp. 208-223. doi : 10.1134/S008154381409020X.

20. Poiseuille J.-L.-M. Recherches expérimentales sur le mouvement des liquides dans les tubes de très-petits diamètres. Paris, Imprimerie Royale, 1844.

21. Batchelor G. K. An Introduction to Fluid Dynamics. Cambridge, Cambridge Univ. Press, 2000, xviii+615 pp. doi : 10.1017/СB09780511800955.

22. Strakhovich K. I. Mekhanika viazkoi zhidkosti. I. Obshchaia chast' [Mechanics of a Viscous Fluids. I. General Part]. Leningrad, Leningrad State Univ., 1940, 201 pp. (In Russian)

23. Szymánski P. Quelques solutions exactes des équations d'hydrodynamique du fluide visqueux dans le cas d'un tube cylindrique, J. Math. Pures Appl., 1932, vol.11, pp. 67108, http://eudml.org/doc/234063. 